\title{
Rituximab Therapy for Treatment of Pemphigus in Southeast Asians
}

\author{
Silada Kanokrungsee $\mathbb{D}^{1,2}$ \\ Tanaporn Anuntrangsee (D) \\ Jutamas Tankunakorn (D) \\ Ploychompoo \\ Srisuwanwattana (iD) 1,3 \\ Poonkiat Suchonwanit (iD) \\ Kumutnart Chanprapaph (D) \\ 'Division of Dermatology, Department of \\ Medicine, Faculty of Medicine, \\ Ramathibodi Hospital, Mahidol \\ University, Bangkok, Thailand; ${ }^{2}$ Graduate \\ School of Srinakharinwirot University, \\ Bangkok, Thailand; ${ }^{3}$ Division of \\ Dermatology, Department of Medicine, \\ Faculty of Medicine, Ramathibodi Chakri \\ Naruebodindra Hospital, Mahidol \\ University, Samut Prakan, Thailand
}

Background: Rituximab provides more effective and less adverse effects than the standard dose of corticosteroids, but evidence on its efficacy and safety in the Thai population is lacking.

Objective: To evaluate the efficacy and safety of rituximab in the treatment of pemphigus and also to determine prognostic factors linked to the treatment outcomes.

Methods: Pemphigus patients who received rituximab from November 2017 to December 2020 were retrospectively reviewed. The outcome was evaluated by using early (end of consolidation phase [ECP]) and late endpoints (complete remission [CR] on/off therapy, immunological remission [IR], and relapse). Adverse events were noted. Prognostic factors associated with remission and relapse were analyzed.

Results: Of 53 pemphigus patients, all attained ECP within 1.61 months. Almost 80\% achieved CR on therapy within a median time of 6.36 months, while $33.9 \%$ reached CR off therapy in 19.74 months. Nearly half had IR within a median time of 6.88 months. Relapse occurred in $33.3 \%$ with a median time of 14 months. In multivariate analysis, receiving rituximab within 12 months of disease duration was more likely to achieve CR off therapy and IR (hazard ratio [HR] 3.79; 95\% confidence interval [CI] 1.38, 10.42; P = 0.01 and $\mathrm{HR}$ $2.74 ; 95 \%$ CI $1.12,6.69 ; \mathrm{P}=0.027$, respectively), whereas older patients and positive antidesmoglein 1 levels at the time of CR were predictive indicators for relapse (HR 1.07; 95\% CI 1.01, 1.13; $\mathrm{P}=0.036$ and $\mathrm{HR} 4.38 ; 95 \%$ CI 1.24, 15.46; $\mathrm{P}=0.022$, respectively). The treatment-related adverse effects occurred in $33.9 \%$.

Conclusion: Rituximab is effective and safe in Thai pemphigus patients. Early administration of rituximab was a predictor of clinical and immunological remission. Older age and persistently positive anti-Dsg1 were correlated with disease relapse.

Keywords: anti-CD20 monoclonal antibody, Asia, autoimmune bullous, pemphigus, rituximab

\section{Introduction}

Pemphigus is a potentially life-threatening chronic autoimmune bullous disease caused by autoantibodies directed against the cell surface protein, antidesmogleins (Dsg). ${ }^{1-3}$ The disease is characterized by mucocutaneous blisters and erosions. ${ }^{4,5}$ Systemic corticosteroids have been considered as the traditional treatment of choice, as they have a high probability of decreasing the mortality rate. ${ }^{6,7}$ However, long-term use at a high dose is often required, resulting in potential adverse effects. ${ }^{8}$ The combination of systemic corticosteroids with other immunosuppressive agents, eg, azathioprine (AZA), mycophenolate mofetil (MMF), cyclophosphamide, cyclosporin, and methotrexate, has been proven to be 
effective, as this approach possesses a steroid-sparing effect and may induce complete clinical remission in pemphigus. $^{9-13}$ Nevertheless, the remission rate is not satisfactory, and a significant number of patients experience relapse following treatments. Intravenous immunoglobulin and plasmapheresis have been employed in the treatment of autoimmune bullous disease, including pemphigus vulgaris; however, the results remain inconsistent. $^{14-17}$

A major development in pemphigus treatment came with the use of rituximab, a chimeric monoclonal antiCD20. Rituximab selectively acts on the CD20expressing B-cells, which are known to secrete antibodies targeting Dsg. This has been an effective treatment for pemphigus and has a steroid-sparing effect. ${ }^{18-20}$ When used as the first-line treatment, rituximab can provide a high clinical remission rate of approximately $70-80 \%$, as reported in studies on Caucasians. ${ }^{21-23}$ A randomized clinical trial showed the superior outcome of rituximab plus short-term corticosteroids, compared to using corticosteroids alone. ${ }^{24}$ As a result, rituximab has been approved by the US Food and Drug Administration and the European Academy of Dermatology and Venereology as a firstline treatment for moderate to severe pemphigus. ${ }^{25,26}$ However, there is limited data regarding its use in Southeast Asia, and a consensus regarding the use of rituximab for Asian pemphigus patients is still lacking. Moreover, rituximab has not been widely used in Thailand, due to its unavailability and financial limitations. Therefore, there is no information on the use of rituximab for Thai pemphigus patients.

Despite the evidence of the efficacy of rituximab, many important questions remain unanswered. The number and timing of a rituximab course; the most effective protocol; whether rituximab should be a first-line agent for pemphigus patients, regardless of disease severity; and the duration of treatment all need to be ascertained. It is also important to know the critical predictors of disease relapse, as this information may determine the benefit of maintenance therapy. Thus far, information on the factors to indicate relapse remains sparse. This study aims to confirm the efficacy of using the recommended outcome parameters, along with the safety of rituximab in the treatment of Thai pemphigus patients. We also intend to determine the prognostic factors associated with remission and relapse.

\section{Materials and Methods}

\section{Study Designs}

This is a retrospective, single-center, cohort study conducted at the Autoimmune Blistering Skin Disease Clinic, Division of Dermatology, Ramathibodi Hospital in Bangkok, Thailand. A uniform patient follow-up protocol was applied. The follow-up visits were scheduled twice monthly after the patients completed the first rituximab cycle, then at 4, 6, and 8 months. Additional visits were scheduled every 2-4 months, depending on each patient's clinical response and/or when needed. On each visit, the patient's clinical data, Autoimmune Bullous Skin Disorder Intensity Score (ABSIS), Pemphigus Disease Area Index score (PDAI), concomitant medication and dosage, and adverse events were noted. Anti-Dsg 1/3 enzyme-linked immunosorbent assays (ELISA) were measured at baseline before initiating rituximab and were reassessed at the $1^{\text {st }}, 2^{\text {nd }}, 4^{\text {th }}, 6^{\text {th }}$, and $8^{\text {th }}$ months after treatment. Our study was approved by the Mahidol University Review Board for Ethics in Human Research, according to Good Clinical Practice Guidelines and the provisions of the World Medical Association's Declaration of Helsinki (MURA2020/1202). All procedures performed in our study involving human participants were in accordance with the institutional research committee. Informed consent was exempted by the board because of the retrospective nature of this study. The data were anonymously analyzed.

\section{Study Population}

The medical records of consecutive pemphigus patients who were treated with rituximab at our clinic from November 2017-December 2020 were reviewed. The inclusion criteria were individuals aged $>15$ years who were diagnosed as pemphigus vulgaris or pemphigus foliaceus, based on the typical clinical presentations, histopathology, immunofluorescence studies, and/or ELISA for the anti-Dsg1 and anti-Dsg3. The exclusion criteria were pemphigus coexisting with other autoimmune diseases, severe liver disease, pregnancy or lactation, incomplete rituximab treatment, and loss to follow-up.

\section{Data Collection}

From the electronic medical record system, we collected the patients' baseline characteristics, disease duration, clinical presentations, and disease-severity assessment using their ABSIS or PDAI score at the beginning of the 
therapy and on every subsequent visit. ${ }^{27,28}$ In accordance with a study by Kim et al, $^{29}$ we categorized patients as being in either an early- or delayed-treatment group. The former group was defined as people who had rituximab treatment within the first 12 months of their disease. The latter group was comprised of those who received rituximab after 12 months of having the disease. Concomitant corticosteroids and adjuvant therapy before and during the rituximab treatment were also noted. Anti-Dsg1 and antiDsg3 antibody values were longitudinally recorded. All patients received rituximab in accordance with either the lymphoma regimen $\left(375 / \mathrm{m}^{2}\right.$ weekly for four weeks) or the rheumatoid arthritis regimen (a 1000-mg infusion two weeks apart). We evaluated the patients at months 1,2 , 4,6 , and 8 after the rituximab infusion and during additional visits. All adverse events were recorded.

\section{Assessment of Treatment Response}

The clinical outcomes of the rituximab treatment were evaluated based on the definitions of the diseaseoutcome parameters in the recent international consensus and comprising the early and late endpoint. ${ }^{30}$ The early endpoint was defined as the end of the consolidation phase (ECP: no new lesions have developed for a minimum of two weeks, and approximately $80 \%$ of the lesions have healed). The late endpoints were determined by; (1) complete remission (CR) on minimal therapy (CR on therapy : absence of new and/or established lesions for at least two months while the patient is receiving prednisolone $\leq 10 \mathrm{mg} / \mathrm{d}$ and/or minimal adjuvant therapy); (2) CR off therapy (no new and/or established lesions while being off all systemic therapy for at least two months); and (3) immunological remission (IR: the time when a positive anti-Dsg1 and/or anti-Dsg3 have or has converted to negative values, which are less than $20 \mathrm{RU}$ [rat units] $/ \mathrm{mL}$ ). In addition, patients who have an extension of established lesions or more than three new lesions per month which do not heal spontaneously within a week after disease control were considered as relapse cases. The number of patients, the median time to achieve each endpoint (which is calculated from the first date of the rituximab infusion), and the median cumulative corticosteroids during each endpoint were also retrieved and analyzed. The median time to relapse was calculated from the first date of $\mathrm{CR}$ on therapy to the date of relapse.

\section{Statistical Analysis}

All data analyses were performed using Stata Version 14.0 (Stata Corp, College Station, TX). Descriptive analysis was conducted to determine the distributions (mean with corresponding standard deviation or median with range and proportion for continuous and categorical variables, respectively). Regarding the treatment endpoint, the survival analysis was estimated by using the Kaplan-Meier method. Univariate analysis was used to investigate the statistical associations between each endpoint and the variables, including one's age at treatment and the baseline PDAI score as continuous data, whereas the pemphigus subtype, dosing regimen, disease duration before rituximab treatment, and the first-line treatment qualified as group data. We also added the level of anti-Dsg1/3 into the equation of predicting factors for relapse. The factors with a trend toward significance in univariate analysis were included in the multivariate logistic regression analysis (adjusting for all relevant confounding factors). Then, the results were reported as the hazard ratio (HR) and corresponding 95\% confidence interval (CI). The Log rank test was used to compare the survival distributions between certain variables of interest. A p-value of less than 0.05 was considered statistically significant.

\section{Results}

\section{Characteristics of the Patients and Their Clinical Course}

Of the 55 pemphigus patients who had been treated with rituximab, one was excluded due to her overlapping with another autoimmune disease, and another person died before completing rituximab therapy. A total of 53 records were enrolled. There were 20 males and 33 females, with a mean age at treatment of $52.91 \pm 15.25$ years. In all, $73.6 \%(\mathrm{n}=39)$ were diagnosed as pemphigus vulgaris and $26.4 \%(\mathrm{n}=14)$ as pemphigus foliaceus, with a median disease duration before the first cycle infusion of 27.43 (0.48-163.15) months. Approximately half of the patients presented with mucocutaneous lesions, while $37.7 \%$ $(\mathrm{n}=20)$ had cutaneous lesions only, and $13.2 \%(\mathrm{n}=7)$ exclusively had mucosal lesions. The median baseline PDAI and ABSIS scores were 13 (3-64) and 8.13 (1.25-50), respectively. The majority of our patients $(79.2 \%, n=42)$ had moderate to severe pemphigus. Twenty-eight percent $(n=15)$ of the individuals were in the early-rituximabtreatment group, while $71.7 \%(\mathrm{n}=37)$ were in the delayedtreatment group. Fifteen percent $(n=8)$ received rituximab 
as the first-line therapy, and the remaining $84.9 \%(n=45)$ received it as a second- or third-line treatment. The baseline anti-Dsg1 and anti-Dsg3 before initiating rituximab were positive in $77.4 \%(n=43)$ and $66 \%(n=35)$ of the patients, respectively. Concomitant prednisolone was administered to $86.8 \%(\mathrm{n}=46)$ of the cases, while $77.4 \%$ $(n=41)$ were given adjuvant drugs, including AZA, MMF, intravenous immunoglobulin, cyclophosphamide, and/or dapsone. The mean follow-up time after the first rituximab infusion was $14.92 \pm 6.47$ months. Seventeen patients (32.1\%) had previous or past hepatitis B virus (HBV) infection (HBsAg-negative and anti-HBc-positive) and received antiviral prophylaxis to prevent $\mathrm{HBV}$ reactivation before they got the rituximab therapy. The clinical and immunological characteristics of our patients are presented in Table 1.

\section{Clinical Response to Rituximab Therapy (Table 2)}

All of the patients achieved ECP within a median duration of 1.61 months after the first infusion. Among those patients, $79.3 \% \quad(n=42)$ reached $C R$ on therapy over a median time of 6.36 months. Almost one-third $(n=18)$ had CR off therapy within 19.74 months. However, 11.3\% $(n=6)$ of the patients who achieved CR on therapy could not discontinue prednisolone because they had steroidwithdrawal symptoms and/or adrenal insufficiency. IR was observed in $47.2 \% \quad(n=25)$ of the cases, with a median time of 6.88 months.

The conversion of anti-Dsg1 from a positive to negative value occurred in $62.8 \%(\mathrm{n}=27)$ of the patients over a median period of 4.13 months, while the conversion of anti-Dsg3 was shown in $53.9 \%$ of the cases within 6.52 months. The mean cumulative doses of prednisolone calculated, from the first day of rituximab infusion to the date that patients achieved outcome parameters, were $560 \mathrm{mg}, 1409.38 \mathrm{mg}, 1770.45 \mathrm{mg}$, and $1346.5 \mathrm{mg}$ for ECP, CR on therapy, CR off therapy, and IR, respectively. All patients gradually tapered their corticosteroids in accordance with the individual's clinical response under an expert dermatologist's management. Of the 42 patients who achieved CR on therapy after the initial cycle, $64.3 \%$ $(\mathrm{n}=27)$ remained in remission, with a mean follow-up period of $14.92 \pm 6.47$ months, whereas $33.3 \%(n=14)$ experienced relapse, with a median duration of 14 months.
Table I Baseline Characteristics in All Patients

\begin{tabular}{|c|c|c|}
\hline Characteristics & \multicolumn{2}{|c|}{$(N=53)$} \\
\hline Gender, n (\%) & \multirow{3}{*}{\multicolumn{2}{|c|}{$\begin{array}{l}20(37.7) \\
33(62.3)\end{array}$}} \\
\hline - Male & & \\
\hline - Female & & \\
\hline \multicolumn{3}{|l|}{ Diagnosis, n (\%) } \\
\hline - Pemphigus vulgaris & \multicolumn{2}{|c|}{$39(73.6)$} \\
\hline - Pemphigus foliaceus & \multicolumn{2}{|c|}{$14(26.4)$} \\
\hline Age at onset, mean, years \pm SD (range) & \multicolumn{2}{|c|}{$48.64 \pm 14.04(17-74)$} \\
\hline Age at treatment, mean, years \pm SD (range) & \multicolumn{2}{|c|}{$52.91 \pm 15.25(17-75)$} \\
\hline BMI, mean, $\mathrm{kg} / \mathrm{m}^{2} \pm \mathrm{SD}$ & \multicolumn{2}{|c|}{$25.86+4.68$} \\
\hline \multicolumn{3}{|l|}{ Underlying disease, $n(\%)$} \\
\hline - Hypertension & \multicolumn{2}{|c|}{$17(32.1)$} \\
\hline - Diabetes mellitus & \multicolumn{2}{|c|}{$14(26.4)$} \\
\hline - Dyslipidemia & \multicolumn{2}{|c|}{$16(30.2)$} \\
\hline - Anemia & \multicolumn{2}{|c|}{$8(15.1)$} \\
\hline - Osteoporosis & \multicolumn{2}{|c|}{$9(17.0)$} \\
\hline - Past HBV infection & \multicolumn{2}{|c|}{$17(32.1)$} \\
\hline $\begin{array}{l}\text { Disease duration before receiving rituximab, } \\
\text { median, months (range) }\end{array}$ & \multicolumn{2}{|c|}{$27.43(0.48-163.15)$} \\
\hline Disease duration before receiving rituximab, $\mathrm{n}$ (\%) & \multirow{3}{*}{\multicolumn{2}{|c|}{$\begin{array}{l}15(28.3) \\
38(71.7)\end{array}$}} \\
\hline$-\leq 12$ months (early treatment) & & \\
\hline$->12$ months (delayed treatment) & & \\
\hline \multicolumn{3}{|l|}{ Lesion, $\mathrm{n}(\%)$} \\
\hline Cutaneous only & \multirow{3}{*}{\multicolumn{2}{|c|}{$\begin{array}{c}20(37.7) \\
6(11.3) \\
14(26.4)\end{array}$}} \\
\hline - Pemphigus vulgaris (cutaneous type) & & \\
\hline - Pemphigus foliaceus & & \\
\hline Mucosal only & \multicolumn{2}{|c|}{$7(13.2)$} \\
\hline Mucocutaneous & \multicolumn{2}{|c|}{$26(49.1)$} \\
\hline Baseline PDAI, median, (range) & \multicolumn{2}{|c|}{$13(3-64)$} \\
\hline Baseline ABSIS, median, (range) & \multicolumn{2}{|c|}{$8.16(1.25-50)$} \\
\hline Severity, n (\%) & \multirow{4}{*}{\multicolumn{2}{|c|}{$\begin{array}{c}11(20.8) \\
33(62.2) \\
9(17.0)\end{array}$}} \\
\hline - Mild & & \\
\hline - Moderate & & \\
\hline - Severe & & \\
\hline Patient category, n (\%) & \multirow{3}{*}{\multicolumn{2}{|c|}{$\begin{array}{c}8(15.1) \\
45(84.9)\end{array}$}} \\
\hline - First-line therapy & & \\
\hline - Second or third-line therapy & & \\
\hline Baseline anti-Dsg antibodies, n (\%) & Anti-Dsgl & Anti-Dsg3 \\
\hline$<20 \mathrm{U} / \mathrm{mL}$ & $12(22.6)$ & $18(33.9)$ \\
\hline $20-100 \mathrm{U} / \mathrm{mL}$ & $10(18.9)$ & $7(13.2)$ \\
\hline $10 \mathrm{I}-200 \mathrm{U} / \mathrm{mL}$ & $6(11.3)$ & II (20.8) \\
\hline$>200 \mathrm{U} / \mathrm{mL}$ & $25(47.2)$ & $17(32.1)$ \\
\hline Follow-up time, mean, month $\pm S D$ (range) & \multicolumn{2}{|c|}{$14.92 \pm 6.47$} \\
\hline
\end{tabular}

Abbreviations: ABSIS, Autoimmune Bullous Skin Disorder Intensity Score; Dsg-I, desmoglein-I; Dsg-3, desmoglein-3; HBV, hepatitis B virus; n, number of patients; PDAI, Pemphigus Disease Area Index; U, unit; SD, standard deviation. 
Table 2 Treatment Response to Rituximab

\begin{tabular}{|c|c|c|c|}
\hline Outcome (n) & All (53) & PV (39) & PF (14) \\
\hline \multicolumn{4}{|l|}{$\mathrm{ECP}$} \\
\hline Number, n (\%) & $53(100)$ & $39(100)$ & $14(100)$ \\
\hline Median time, month & 1.61 & 1.61 & 1.61 \\
\hline Cumulative prednisolone, median, mg (range) & $560(0-2100)$ & $580(0-2100)$ & $389.35(0-1605)$ \\
\hline \multicolumn{4}{|l|}{$C R$ on therapy } \\
\hline Number, n (\%) & $42(79.3)$ & $31(79.5)$ & II (78.6) \\
\hline Median time, month & 6.36 & 6.36 & 5.28 \\
\hline Cumulative prednisolone, median, mg (range) & $1409.38(0-4065)$ & $1470(0-4065)$ & $1045.9(0-3190)$ \\
\hline \multicolumn{4}{|l|}{ CR off therapy } \\
\hline Number, n (\%) & $18(34)$ & 14 (35.9) & $4(28.6)$ \\
\hline Median time, month & 19.74 & 19.74 & - \\
\hline Cumulative prednisolone, median, mg (range) & $1770.45(0-5075)$ & $1803.58(0-5075)$ & $|4| 6 . \mid(0-3 \mid 49.6)$ \\
\hline \multicolumn{4}{|l|}{ Immunological remission (all) } \\
\hline Number, n (\%) & $25(47.2)$ & $20(5 \mid .3)$ & $5(35.7)$ \\
\hline Median time, month & 6.88 & 6.52 & - \\
\hline Cumulative prednisolone, median, mg (range) & $1346.5(0-3555)$ & I $347.63(0-3555)$ & $1045.9(444.3-3190)$ \\
\hline \multicolumn{4}{|l|}{ Immunological remission (anti- Dsg-I) } \\
\hline Number, n (\%) & $27 / 43(62.8)$ & $22 / 29(75.9)$ & $5(35.7)$ \\
\hline Median time, month & 4.13 & 3.41 & - \\
\hline \multicolumn{4}{|l|}{ Immunological remission (anti-Dsg-3) } \\
\hline Number, n (\%) & - & $21(53.9)$ & - \\
\hline Median time, month & - & 6.52 & - \\
\hline \multicolumn{4}{|l|}{ Relapse } \\
\hline Number, n (\%) & $14(33.3)$ & $10(32.3)$ & $4(36.4)$ \\
\hline Median time, month & 14 & 14 & 20.20 \\
\hline
\end{tabular}

Abbreviations: Dsg-I, desmoglein-I; Dsg-3, desmoglein-3; n, number of patients; PF, pemphigus foliaceus; PV, pemphigus vulgaris.

\section{Assessment of the Prognostic Factors (Table 3)}

\section{Factors Associated with CR}

In both the univariate and multivariate analyses, variables including the patient's clinical characteristic, gender, age, pemphigus subtype, baseline PDAI score, baseline antiDsg1, anti-Dsg3, and concurrent immunosuppressants/ immunomodulators (ie, AZA, MMF, cyclophosphamide, dapsone) were not significantly related to CR. Likewise, there were no differences between the rheumatoid and lymphoma regimens $(P=0.110)$. However, univariate analysis showed that patients who had an early administration of rituximab, in contrast to the delayed-treatment group, were more likely to achieve $\mathrm{CR}$ on and off therapy by 2.22 -fold (HR 2.22; 95\% CI 1.12, 4.41; $\mathrm{P}=0.023$ ) and 3.14-fold (HR 3.14; 95\% CI 1.20, 8.19; $\mathrm{P}=0.020$ ), respectively. Moreover, the early-treatment group required a shorter median duration to reach CR on therapy (5.77 vs
7.31 months) and CR off therapy (14.13 months vs unable to calculate), as shown in Figure 1. A multivariate analysis repeatedly showed that patients with an early rituximab administration were more likely to reach CR off therapy, with statistical significance (HR 3.79; 95\% CI 1.38, 10.42; $\mathrm{P}=0.010$ ). Pemphigus patients who received rituximab as the first-line therapy also achieved $\mathrm{CR}$ on therapy more than those who were given it as a second- or third-line treatment by 2.57 -fold (HR 2.57; 95\% CI 1.10, 6.04; $\mathrm{P}=$ 0.030). The first-line treatment group reached CR on therapy within a shorter median time than patients receiving rituximab as a second- or third-line approach (5.05 vs 6.66 months), as shown in Figure 2. However, using rituximab as the first-line therapy did not have a correlation with a higher chance of attaining $C R$ off therapy $(P=0.819)$.

\section{Factors Associated with IR}

In the univariate analysis, a statistical association was found between early treatment and IR. The early- 
Table 3 Factors Associated with Disease Outcome

\begin{tabular}{|c|c|c|c|c|}
\hline \multirow{2}{*}{$\begin{array}{l}\text { Clinical Remission on Therapy } \\
\text { Factor }\end{array}$} & \multicolumn{2}{|c|}{ Univariate } & \multicolumn{2}{|c|}{ Multivariate } \\
\hline & Hazard Ratio $(95 \% \mathrm{Cl})$ & P-value & Hazard Ratio $(95 \% \mathrm{Cl})$ & P-value \\
\hline $\begin{array}{l}\text { Diagnosis } \\
\text { - Pemphigus vulgaris } \\
\text { - Pemphigus foliaceus }\end{array}$ & $\begin{array}{l}\text { I.0I }(0.50,2.0 \mathrm{I}) \\
\text { I [Reference] }\end{array}$ & 0.987 & $0.75(0.32,1.76)$ & 0.508 \\
\hline Age at treatment & $1.00(0.98,1.02)$ & 0.995 & $1.00(0.98,1.03)$ & 0.811 \\
\hline PDAI baseline & $1.00(0.98,1.03)$ & 0.917 & $1.00(0.97,1.03)$ & 0.886 \\
\hline $\begin{array}{l}\text { Immunosuppressants/immunomodulators } \\
\text { - Yes } \\
\text { - No }\end{array}$ & $\begin{array}{l}0.77(0.37,1.64) \\
\text { I [Reference] }\end{array}$ & 0.509 & - & - \\
\hline $\begin{array}{l}\text { Baseline anti-Dsgl } \\
\text { - }<20 \mathrm{U} / \mathrm{mL} \\
\text { - } 20-200 \mathrm{U} / \mathrm{mL} \\
\text { - }>200 \mathrm{U} / \mathrm{mL}\end{array}$ & $\begin{array}{c}\text { I [Reference] } \\
0.66(0.30,1.47) \\
0.63(0.29,1.35)\end{array}$ & $\begin{array}{l}0.310 \\
0.232\end{array}$ & - & \\
\hline $\begin{array}{l}\text { Baseline anti-Dsg3 } \\
\text { - }<20 \mathrm{U} / \mathrm{mL} \\
\text { - } 20-200 \mathrm{U} / \mathrm{mL} \\
\text { - }>200 \mathrm{U} / \mathrm{mL}\end{array}$ & $\begin{array}{c}\text { I [Reference] } \\
\text { I.05 }(0.5 \mathrm{I}, 2.19) \\
0.32(0.43,1.97)\end{array}$ & $\begin{array}{l}0.891 \\
0.837\end{array}$ & - & \\
\hline $\begin{array}{l}\text { Regimen } \\
\text { - Rheumatoid arthritis } \\
\text { - Lymphoma }\end{array}$ & $\begin{array}{c}2.16(0.84,5.56) \\
\text { I [Reference] }\end{array}$ & 0.110 & $2.07(0.75,5.76)$ & 0.162 \\
\hline $\begin{array}{l}\text { Disease duration } \\
\text { - Early treatment ( } \leq 12 \text { months) } \\
\text { - Delayed treatment (>12 months) }\end{array}$ & $\begin{array}{c}2.22(\mathrm{I} .12,4.4 \mathrm{I}) \\
\mathrm{I}[\text { Reference }]\end{array}$ & $0.023^{*}$ & I.78 $(0.74,4.27)$ & 0.198 \\
\hline $\begin{array}{l}\text { Administration } \\
\text { - First-line therapy } \\
\text { - Second- or third-line therapy }\end{array}$ & $\begin{array}{c}2.57(1.10,6.04) \\
\text { I [Reference] }\end{array}$ & $0.030^{*}$ & $\mathrm{I} .63(0.58,4.63)$ & 0.355 \\
\hline Clinical Remission off Therapy & Univari & & Multivar & \\
\hline Factor & Hazard Ratio $(95 \% \mathrm{Cl})$ & P-value & Hazard Ratio (95\% Cl) & P-value \\
\hline $\begin{array}{l}\text { Diagnosis } \\
\text { - Pemphigus vulgaris } \\
\text { - Pemphigus foliaceus }\end{array}$ & $\begin{array}{l}\text { I.33 }(0.43,4.16) \\
\text { I [Reference] }\end{array}$ & 0.620 & $\mathrm{I} .66(0.44,6.20)$ & 0.451 \\
\hline Age at treatment & I.0I $(0.98,1.04)$ & 0.554 & $1.02(0.98,1.06)$ & 0.448 \\
\hline PDAI baseline & $0.98(0.92,1.03)$ & 0.418 & $0.96(0.90,1.02)$ & 0.174 \\
\hline $\begin{array}{l}\text { Immunosuppressants/immunomodulators } \\
\text { - Yes } \\
\text { - No }\end{array}$ & $\begin{array}{l}\text { I.86 }(0.43,8.12) \\
\text { I [Reference] }\end{array}$ & 0.407 & - & - \\
\hline $\begin{array}{l}\text { Baseline anti-Dsgl } \\
\text { - }<20 \mathrm{U} / \mathrm{mL} \\
\text { - } 20-200 \mathrm{U} / \mathrm{mL} \\
\text { - }>200 \mathrm{U} / \mathrm{mL}\end{array}$ & $\begin{array}{c}\text { I [Reference] } \\
0.68(0.19,2.35) \\
0.80(0.24,2.59)\end{array}$ & $\begin{array}{l}0.538 \\
0.705\end{array}$ & - & - \\
\hline
\end{tabular}


Table 3 (Continued).

\begin{tabular}{|c|c|c|c|c|}
\hline $\begin{array}{l}\text { Baseline anti-Dsg3 } \\
\text { - }<20 \mathrm{U} / \mathrm{mL} \\
\text { - } 20-200 \mathrm{U} / \mathrm{mL} \\
\text { - }>200 \mathrm{U} / \mathrm{mL}\end{array}$ & $\begin{array}{c}\text { I }[\text { Reference }] \\
\text { I.02 }(0.37,2.86) \\
0.42(0.11,1.65)\end{array}$ & $\begin{array}{l}0.965 \\
0.216\end{array}$ & - & - \\
\hline $\begin{array}{l}\text { Disease duration } \\
\text { - Early treatment ( } \leq 12 \text { months) } \\
\text { - Delayed treatment (>12 months) }\end{array}$ & $\begin{array}{c}3.14(1.20,8.19) \\
\text { I [Reference] }\end{array}$ & $0.020 *$ & $3.79(1.38,10.42)$ & $0.010^{*}$ \\
\hline $\begin{array}{l}\text { Administration } \\
\text { - First-line therapy } \\
\text { - Second- or third-line therapy }\end{array}$ & $\begin{array}{c}1.16(0.33,4.00) \\
\text { I [Reference] }\end{array}$ & 0.819 & - & - \\
\hline Immunological Remission & \multicolumn{2}{|c|}{ Univariate } & \multicolumn{2}{|c|}{ Multivariate } \\
\hline Factor & Hazard Ratio ( $95 \% \mathrm{Cl})$ & P-value & Hazard Ratio (95\% Cl) & P-value \\
\hline $\begin{array}{l}\text { Diagnosis } \\
\text { - Pemphigus vulgaris } \\
\text { - Pemphigus foliaceus }\end{array}$ & $\begin{array}{c}\text { I.54 }(0.58,4.10) \\
\text { I [Reference] }\end{array}$ & 0.388 & $1.26(0.40,3.98)$ & 0.696 \\
\hline Age at treatment & $1.00(0.97,1.03)$ & 0.928 & $1.0(0.97,1.03)$ & 0.848 \\
\hline PDAI baseline & $1.03(1.01,1.05)$ & $0.029 *$ & $1.01(0.99,1.04)$ & 0.296 \\
\hline $\begin{array}{l}\text { Immunosuppressants/immunomodulators } \\
\text { - Yes } \\
\text { - No }\end{array}$ & $\begin{array}{c}0.88(0.35,2.22) \\
\text { I [Reference] }\end{array}$ & 0.793 & - & - \\
\hline $\begin{array}{l}\text { Baseline anti-Dsgl } \\
\text { - }<20 \mathrm{U} / \mathrm{mL} \\
\text { - } 20-200 \mathrm{U} / \mathrm{mL} \\
\text { - }>200 \mathrm{U} / \mathrm{mL}\end{array}$ & $\begin{array}{c}\text { I [Reference] } \\
0.75(0.25,2.22) \\
0.97(0.36,2.59)\end{array}$ & $\begin{array}{l}0.599 \\
0.956\end{array}$ & - & - \\
\hline $\begin{array}{l}\text { Baseline anti-Dsg3 } \\
\text { - }<20 \mathrm{U} / \mathrm{mL} \\
\text { - } 20-200 \mathrm{U} / \mathrm{mL} \\
\text { - }>200 \mathrm{U} / \mathrm{mL}\end{array}$ & $\begin{array}{c}\text { I [Reference] } \\
2.25(0.90,5.61) \\
0.45(0.13,1.53)\end{array}$ & $\begin{array}{l}0.081 \\
0.198\end{array}$ & - & - \\
\hline $\begin{array}{l}\text { Regimen } \\
\text { - Lymphoma dose } \\
\text { - Rheumatoid arthritis dose }\end{array}$ & $\begin{array}{c}\text { I [Reference] } \\
0.83(0.28,2.4 \mathrm{I})\end{array}$ & 0.726 & $0.61(0.19,1.98)$ & 0.411 \\
\hline $\begin{array}{l}\text { Disease duration } \\
\text { - Early treatment ( } \leq 12 \text { months) } \\
\text { - Delayed treatment (>12 months) }\end{array}$ & $\begin{array}{c}2.93(1.32,6.50) \\
\text { I [Reference] }\end{array}$ & $0.008^{*}$ & $2.74(1.12,6.69)$ & $0.027^{*}$ \\
\hline $\begin{array}{l}\text { Administration } \\
\text { - First-line therapy } \\
\text { - Second- or third-line therapy }\end{array}$ & $\begin{array}{c}1.92(0.72,5.16) \\
\text { I [Reference] }\end{array}$ & 0.195 & - & - \\
\hline Relapse & \multicolumn{2}{|c|}{ Univariate } & \multicolumn{2}{|c|}{ Multivariate } \\
\hline Factor & Hazard Ratio (95\% Cl) & P-value & Hazard Ratio (95\% Cl) & P-value \\
\hline $\begin{array}{l}\text { Diagnosis } \\
\text { - Pemphigus vulgaris } \\
\text { - Pemphigus foliaceus }\end{array}$ & $\begin{array}{c}0.90(0.25,3.28) \\
\text { I [Reference] }\end{array}$ & 0.872 & $1.95(0.37,10.42)$ & 0.434 \\
\hline
\end{tabular}


Table 3 (Continued).

\begin{tabular}{|c|c|c|c|c|}
\hline Age at treatment & $1.05(1.01,1.10)$ & $0.040^{*}$ & $1.07(1.01,1.13)$ & $0.036^{*}$ \\
\hline PDAI baseline & $1.03(0.96,1.11)$ & 0.357 & $1.04(0.97,1.12)$ & 0.240 \\
\hline $\begin{array}{l}\text { Immunosuppressants/immunomodulators } \\
\text { - Yes } \\
\text { - No }\end{array}$ & $\begin{array}{c}0.53(0.16,1.73) \\
\text { I [Reference] }\end{array}$ & 0.290 & - & - \\
\hline $\begin{array}{l}\text { Regimen } \\
\text { - Lymphoma dose } \\
\text { - Rheumatoid arthritis dose }\end{array}$ & $\begin{array}{c}\text { I [Reference] } \\
3.42(0.43,27.19)\end{array}$ & 0.244 & $2.72(0.31,23.68)$ & 0.365 \\
\hline $\begin{array}{l}\text { Anti-Dsg I level at clinical remission } \\
\text { - Negative }(<20 \mathrm{U} / \mathrm{mL}) \\
\text { - Positive }(\geq 20 \mathrm{U} / \mathrm{mL})\end{array}$ & $\begin{array}{c}\text { I [Reference] } \\
3.03(0.95,9.67)\end{array}$ & 0.061 & $4.38(1.24,15.46)$ & $0.022 *$ \\
\hline $\begin{array}{l}\text { Anti-Dsg } 3 \text { level at clinical remission } \\
\text { - Negative }(<20 \mathrm{U} / \mathrm{mL}) \\
\text { - Positive }(\geq 20 \mathrm{U} / \mathrm{mL})\end{array}$ & $\begin{array}{c}\text { I [Reference] } \\
\text { I.I0 }(0.36,3.38)\end{array}$ & 0.869 & - & - \\
\hline $\begin{array}{l}\text { Disease duration } \\
\text { - Early treatment ( } \leq 12 \text { months) } \\
\text { - Delayed treatment (>12 months) }\end{array}$ & $\begin{array}{c}0.8 \mathrm{I}(0.22,2.98) \\
\text { I [Reference] }\end{array}$ & 0.746 & - & - \\
\hline $\begin{array}{l}\text { Administration } \\
\text { - First-line therapy } \\
\text { - Second- or third-line therapy }\end{array}$ & $\begin{array}{c}0.80(1.78,3.64) \\
\text { I [Reference] }\end{array}$ & 0.777 & - & - \\
\hline
\end{tabular}

Note: *P-value $<0.05$, indicating a statistically significant difference.

Abbreviations: $\mathrm{Cl}$, confidence interval; Dsg-I, desmoglein-I; Dsg-3, desmoglein-3; U, unit.

treatment group was more likely to achieve IR, with an HR of 2.93 (95\% CI 1.32, 6.50; $\mathrm{P}=0.008)$, compared to the delayed-treatment group. After adjusting for all the confounding factors, the results continued to show that early treatment was a positive predictor for IR (HR 2.74; $95 \%$ CI 1.12, 6.69; $\mathrm{P}=0.027)$. The median time required to reach IR was also shorter in the early-treatment group (Figure 3). Although the rate of achieving IR was correlated with the baseline PDAI score in the univariable analysis (HR 1.03; 95\% CI 1.01, 1.05; P = 0.029), it was no longer significant after adjusting for all the relevant confounding factors in the multivariate analysis.

\section{Factors Associated with Disease Relapse}

The univariate analysis revealed that the age at treatment was a predictor for disease relapse. We found a $5 \%$ increase in the relapse rate, in association with a oneyear age increase (HR 1.05; 95\% 1.01, 1.10; $\mathrm{P}=0.040$ ). Moreover, there was a trend towards a significant association between the anti-Dsg1 level when patients achieved $\mathrm{CR}$ and the rate of relapse. Patients with a positive antiDsg1 level at the time of CR had a 3.03-times increase in their relapse rate, compared to those with negative values
(95\% CI $0.95,9.67 ; \mathrm{P}=0.061)$. The median duration to relapse among anti-Dsg1-negative patients appeared to be longer than those with positive values (20.20 vs 9.02 months), as is shown in Figure 4. After a multivariable analysis was performed, the results repeatedly showed that a higher rate of relapse occurred among those of increasing age (HR 1.07; 95\% CI 1.01, 1.13; $\mathrm{P}=0.036$ ). Likewise, a negative anti-Dsg1 level at the time of CR was a significant protective factor for disease relapse, whereas persistently positive anti-Dsg1 patients were 4.38 times more likely to relapse at any time point, compared to those with negative values (95\% CI 1.24, 15.46; $\mathrm{P}=0.022$ ). Other factors, such as gender, pemphigus subtype, severity score, and dose regimen were not associated with relapse.

\section{Adverse Effects}

A total of 21 adverse effects in 18 patients (33.9\%) were noted. An immediate infusion reaction occurred in 13.21\% $(n=7)$ of the patients. Such reactions included urticaria, tachycardia, tachypnea, and/or abdominal discomfort (eg, nausea, vomiting). Those patients were managed by 

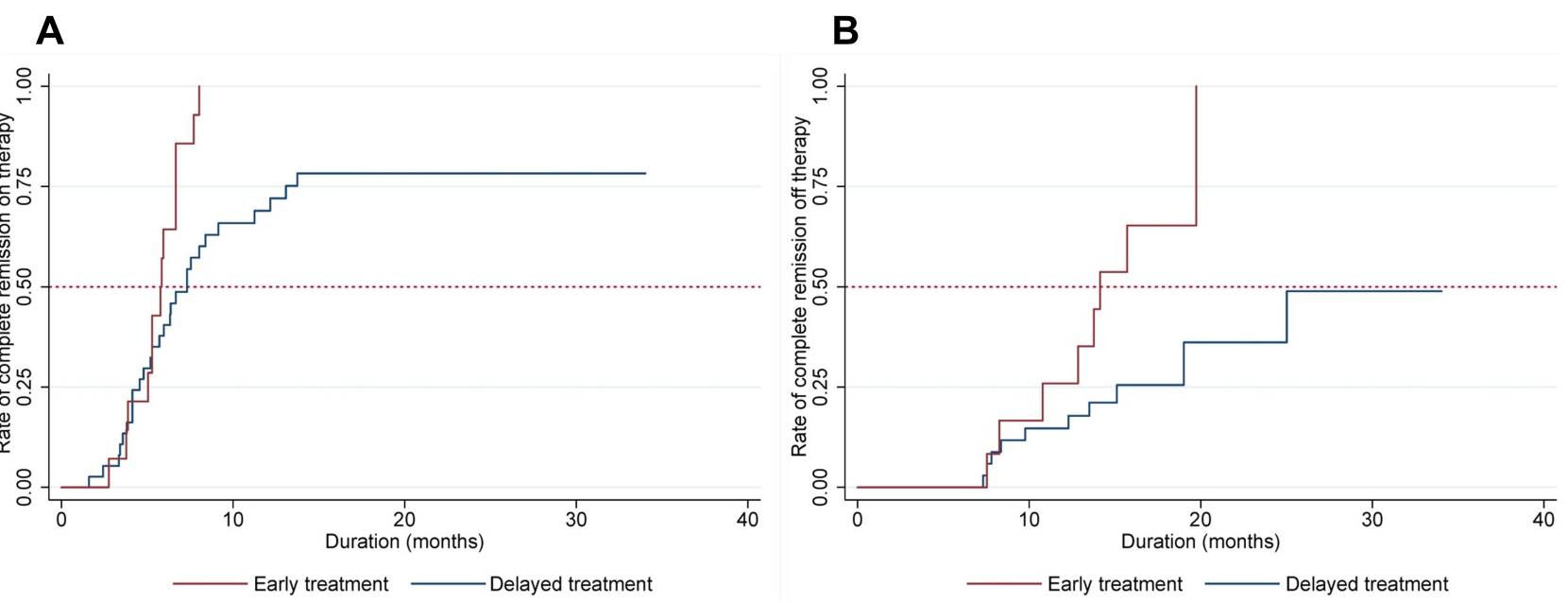

Figure I (A) Kaplan-Meier plot showing the rate of complete remission on therapy between early and delayed treatment. (B) Kaplan-Meier plot showing the rate of complete remission off therapy between early and delayed treatment.

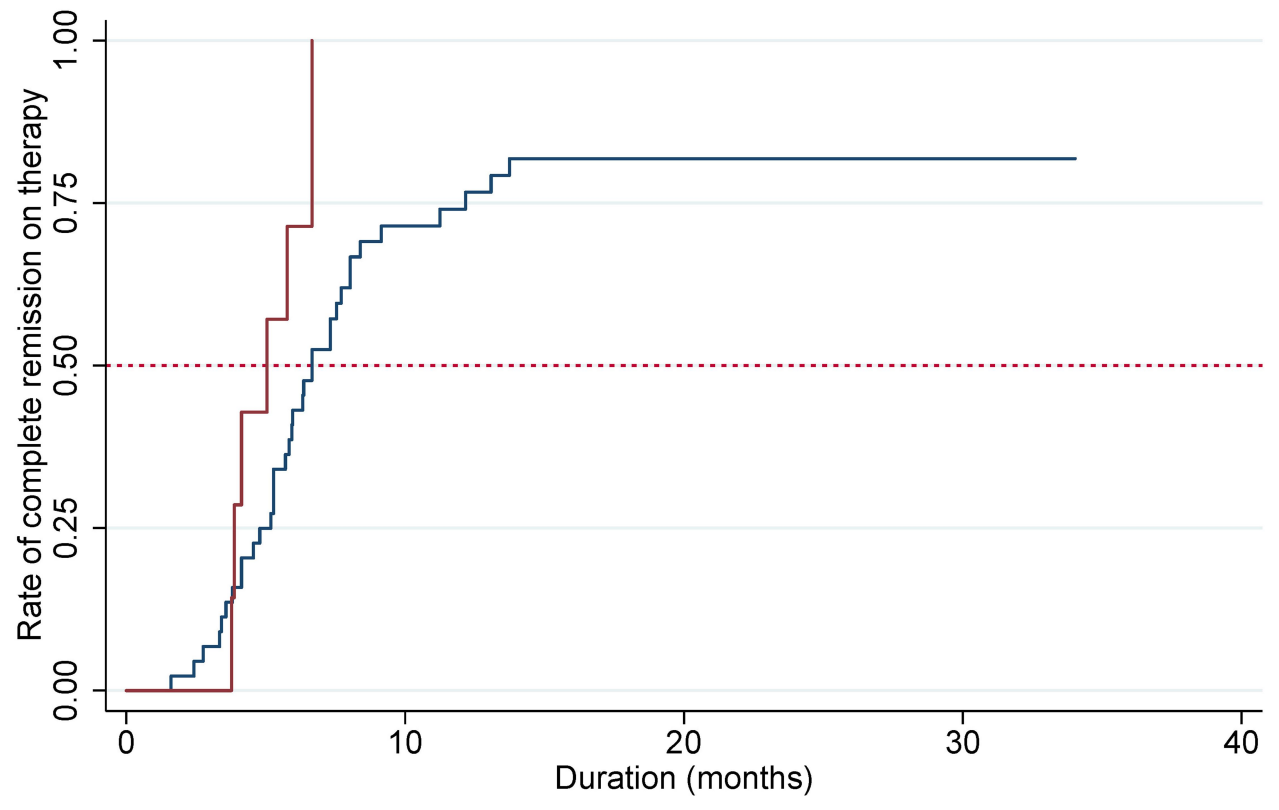

First-line therapy

Second or third-line therapy

Figure 2 Kaplan-Meier plot showing the rate of complete remission on therapy between first-line and second or third-line therapy.

slowing down the infusion rate, and they did not develop further complications. Most events occurred at the individual's first exposure to rituximab. One patient developed grade II reactions to rituximab at his fourth infusion, and he was subsequently admitted for rituximab desensitization. Infectious complications occurred in $16.98 \%(\mathrm{n}=12)$ of the cases. Four people (7.54\%) developed serious infection requiring hospitalization (ie, cytomegalovirus viremia $[\mathrm{n}=1]$, disseminated herpes zoster $[\mathrm{n}=1]$, Pneumocystis jiroveci pneumonia $[\mathrm{n}=1]$, and bacterial sepsis $[\mathrm{n}=1]$ ). Other less-serious infectious events were herpes simplex infection $(n=3)$, oral candidiasis $(n=2)$, herpes zoster infection $(\mathrm{n}=1)$, cystitis $(\mathrm{n}=1)$, and tinea corporis $(\mathrm{n}=1)$. Leukopenia was observed in one $(1.89 \%)$ of the cases, which may be attributable to rituximab and/or its combination with MMF. There was no evidence of HBV-flaring during or after the rituximab treatment. No deaths occurred during the follow-up period. 


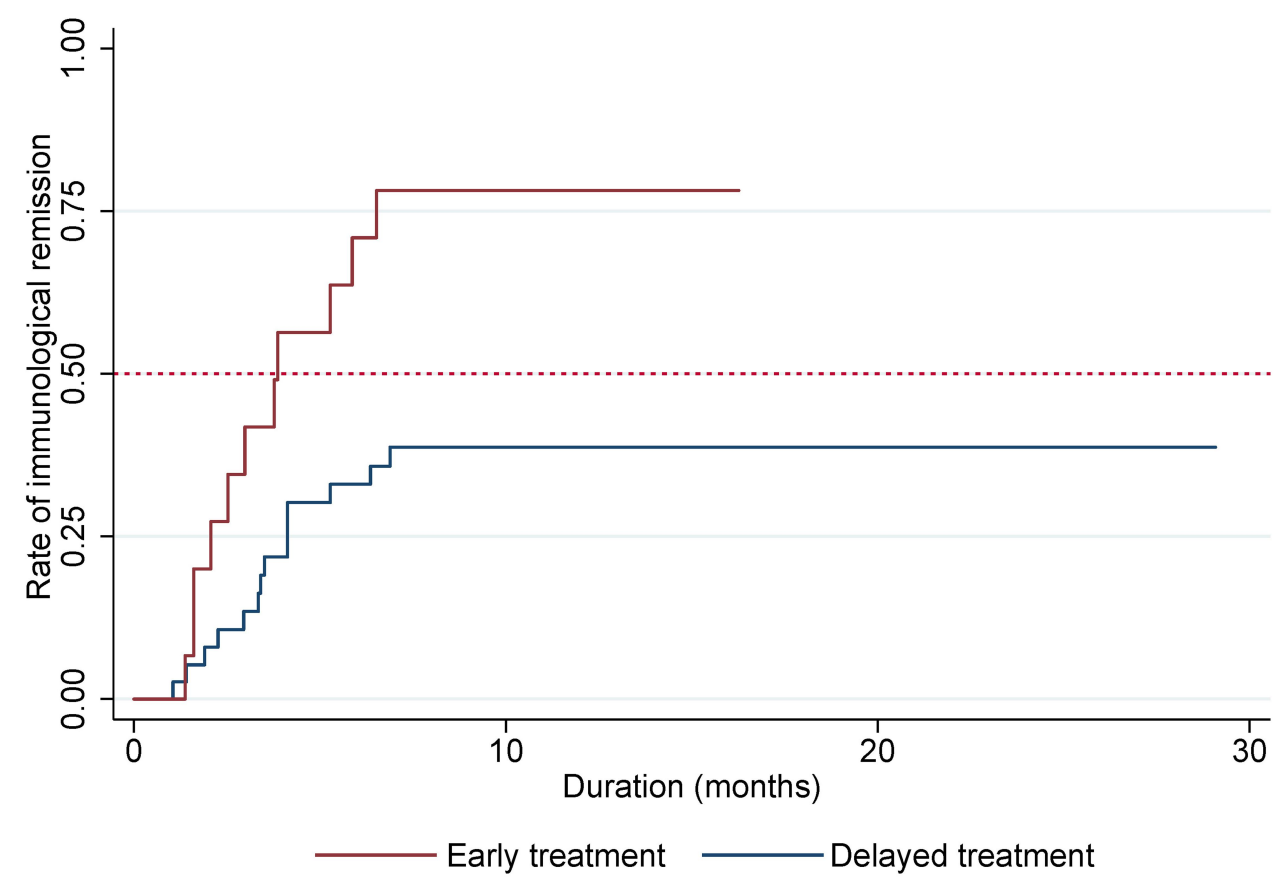

Figure 3 Kaplan-Meier plot showing the rate of immunological remission between early and delayed treatment.

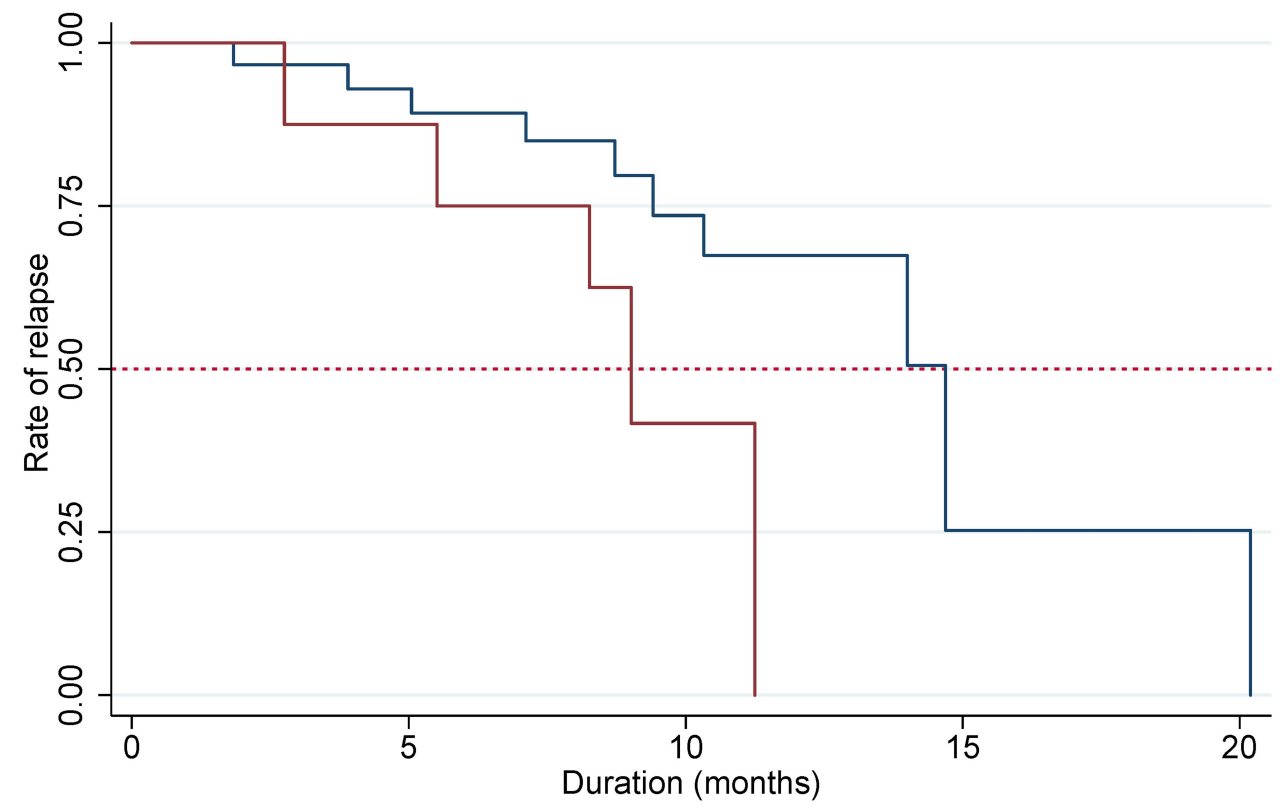

Negative anti-Dsg1 level $\longrightarrow$ Positive anti-Dsg1 level

Figure 4 Kaplan-Meier plot showing the rate of relapse between negative and positive anti-Dsgl level at complete remission.

\section{Discussion}

Rituximab has currently been used for the treatment of pemphigus patients and has had a favorable outcome. $^{21-24,31}$ Ours is the first study on the efficacy and safety of rituximab used for Southeast Asian patients with pemphigus. This study confirms the efficacy of rituximab for pemphigus. Not only does rituximab induce a high remission rate (up to $80 \%$ ); it provides a low 
disease relapse. These promising results may partially counterbalance the costs of managing disease flare-ups and the side effects that patients experience from conventional therapy. ${ }^{32}$ All of our patients had clinical improvement in just over a month, and $79.3 \%$ achieved CR on therapy within 6.36 months following the first induction. Our results were comparable to a recent retrospective study by Sharma et al, in which $83.6 \%$ of their pemphigus patients had CR after rituximab, with a mean duration of $4.8 \pm 2.52$ months. $^{33}$ The rate of CR on therapy in the literature has ranged from $50-89.5 \%$ following the first rituximab infusion, which is tantamount to ours. ${ }^{24,34-36}$ Our data show that CR off therapy occurred in 34\% of the patients within 19.74 months. That rate is rather inferior to the figures in previous reports, where approximately $36.6-89.5 \%$ of the cases achieved CR off therapy. ${ }^{36-38}$ A valid explanation is that, prior to receiving rituximab, the majority of our patients $(84.9 \%)$ had a recalcitrant and relapsing disease. Balighi et al also noted that only $36.6 \%$ of patients who were given rituximab as a second- or thirdline treatment reached CR off therapy. ${ }^{38}$ Moreover, an additional $11.3 \%$ of our patients would presumably have reached $\mathrm{CR}$ off therapy, but had to continue low-dose corticosteroids because they developed steroidwithdrawal symptoms and/or adrenal insufficiency.

Identifying factors associated with $\mathrm{CR}$ after rituximab therapy is crucial in the clinical setting. The remission rate in this study was influenced by two factors: the disease's duration before the rituximab infusion, and rituximab administration as a first-line treatment. To the best of our knowledge, this is the first study to report that early rituximab administration (within 12 months) can increase not only the rate of complete $\mathrm{CR}$ but also the IR. Moreover, patients in the early- treatment group required a shorter time to achieve CR on and off therapy, which led to fewer corticosteroids exposure and complications. Similar to prior studies, we found that the early administration of rituximab resulted in better outcomes, including a higher remission rate, a longer disease-free period, and a lower rate of disease relapse. ${ }^{38-40}$ Our data show that pemphigus patients who received an early administration of rituximab also achieved a higher rate of IR. Persistent anti-Dsg B-cell clones have been documented to appear over many years in patients with active and relapsing disease even in rituximab-treated patients. ${ }^{41}$ Early rituximab treatment may eliminate these sets of the lineage leading to clinical response, as well as prohibiting future
B-cell repopulation. Future studies to determine the true nature of recalcitrant pemphigus are needed.

The second prognostic factor for CR was rituximab given as the first-line treatment. In the majority of studies reported in the literature, rituximab was used as adjuvant therapy for refractory pemphigus patients. A few studies have demonstrated its efficacy as a first-line treatment. $^{24,29,42-45}$ Joly et al conducted an open-label, randomized trial and revealed that the first-line use of rituximab, in combination with short-term prednisolone, resulted in a higher probability (almost three times) of achieving CR off therapy, in contrast to using prednisolone alone. ${ }^{24}$ Vinay et al have also shown that the first-line use of rituximab resulted in a statistically significant rate of achieving CR off therapy, compared to administering it following other treatments. ${ }^{31}$ In the present study, rituximab administered as the first-line therapy was significantly associated with achieving $\mathrm{CR}$ on, but not $\mathrm{CR}$ off therapy and IR. The small number of patients in the firstline group (15.1\%) may likely have led to an insignificant statistical power in determining these differences. The US and European guidelines recommend rituximab as the first-line modality for pemphigus, owing to a high probability of CR. ${ }^{19,24,31,36,46}$ However, its application as the first-line treatment in Southeast Asian pemphigus patients requires further evidence. Moreover, concerns are increasing because rituximab is not readily available or affordable in every hospital setting.

The data regarding which rituximab dosing regimen has optimal benefits in controlling pemphigus remain inconsistent. While Kushner et al reported that the lymphoma regimen has superior efficacy in achieving deeper B-cell depletion in secondary lymphoid tissues than does the rheumatoid regimen, ${ }^{35}$ many other studies, including ours, have not shown the benefits of one regimen over another. $^{35,47,48}$

It is unclear if rituximab can prevent disease relapse. Identifying the predictive factors to determine who may experience relapse remains crucial, as they may establish whether or not the maintenance of rituximab is required. To date, this issue has not been well-documented or studied. Although 73.6\% of our patients achieved CR at the end of this study, $26.4 \%$ of them experienced a relapse, with a median time of 14 months after complete clinical remission. Documents on the relapse rate and the time to relapse have varied in the literature, occurring in 23.7$76.5 \%$ after the first infusion and within six to 24 months. ${ }^{24,29,33,37,38}$ This large discrepancy may be due to 
the differences in the studied population, the patientselection process, the disease's severity, the duration of the disease, and the follow-up period. ${ }^{24,29,33,35,49}$ The first clinical predictor for relapse in our study was the person's age at treatment. A previous report revealed that the older patients were more likely to achieve a clinical response as a result of their immune weakness. ${ }^{35}$ On the contrary, we found that the patient's age at the disease's onset did not influence his or her clinical response. Our data further demonstrated that elderly patients have a higher chance of relapse. The mechanisms underlying the cellular alterations in aging are unclear. In one animal study, immunosenescence in the B-lineage was shown to be reversible, and, after B-cell depletion, it can rejuvenate and enhance one's immune competence. ${ }^{50}$ However, the definite mechanism behind how advanced age influences the disease's relapse remains to be validated by further studies.

Our data also showed that IR (anti-Dsg1) was another predictor of disease relapse. The levels of the anti-Dsg autoantibodies correlated with the disease activity. ${ }^{51}$ Relapse after rituximab treatment has been linked to B-cells repopulation corresponding to the level of autoantibodies, particularly anti-Dsg $1 .^{52}$ Studies have demonstrated that patients with recurrent disease experience a reappearance of the exact same autoreactive B-cell clone. ${ }^{41}$ Previous studies have also shown that anti-Dsg1 was a reliable predictor of disease relapse following rituximab infusion, especially among patients with cutaneous involvement. ${ }^{53,54}$ In addition, a lower anti-Dsg1 titer at the baseline demonstrated a negative correlation with timing for relapse. ${ }^{55}$ Recently, Mignard et al observed that patients with relapse had higher anti-Dsg1 and anti-Dsg3 antibody levels (at month 3), compared to those who had no relapse. ${ }^{56}$ Our results correspond with the literature. ${ }^{56}$ We found that patients with undetectable anti-Dsg1 at the time of CR had a four-times-lower tendency for relapse, compared to patients with persistently positive values. The median time to relapse was also longer in patients with negative anti-Dsg1 at CR than among those without (20 months vs 9 months). We further demonstrated that the duration of anti Dsg1 conversion (positive to negative) was shorter than the time required for CR (4.13 vs 6.36 months). The level of anti-Dsg1 is expected to be negative before clinical remission because rituximab causes prompt and nearly complete depletion of peripheral CD20+ cells, as well as inhibition of antibody production. After treatment, persistently positive anti-Dsg1 may suggest a subsequent relapse due to B-cell repopulation. ${ }^{41}$
Notably, we highlighted the importance of IR as a predictor of disease relapse. Therefore, we suggest that the anti-Dsg1 levels should be measured at the time of complete CR. If they remain positive, close monitoring during the first 12 months of follow-up is recommended.

Regarding the safety of rituximab, the total adverse reaction previously reported had ranged from $4.8-35 \%$ for serious adverse events (SAEs) to $5.5-16 \%$ for an infusion reaction. ${ }^{37,40,57-59}$ Our results were in line with those of earlier studies: $7.5 \%$ of rituximab-treated patients experienced SAEs, while $13.2 \%$ of them had infusion reactions. We did not come across any fatal side effects, which have previously been reported to occur in 1.6$12.5 \%$ of the cases. ${ }^{57}$ Moreover, it is important to note that antiviral prophylaxis has helped protect our patients from HBV reactivation.

There are several limitations to our study. First, the retrospective nature of the study may have led to some incomplete data. However, all patients were treated in the same clinic by the same expert dermatologist, and a uniform monitoring and follow-up protocol were applied. Our study lacked comprehensive profiles regarding B-cell depletion by flow cytometric assay because they are not routinely assessed in our institute. Secondly, a selection bias may have occurred, as the study was performed in a tertiary-care referral center. Also, several cases were initially managed by community dermatologists, so we were unable to determine many parameters from the disease's onset. Finally, our sample size was relatively small. The cost of rituximab was a major factor in limiting the number of patients in this study.

\section{Conclusion}

We confirm the efficacy and safety of rituximab for our Thai pemphigus patients. Early rituximab administration within 12 months was a predictor of both CR and IR. Older patients and those with a persistently positive serum anti-Dsg1 were associated with disease relapse. Finally, antiviral prophylaxis helped protect patients from HBV reactivation.

\section{Acknowledgments}

The study was supported by the Division of Dermatology and the Clinical Research Unit, all form Faculty of Medicine, Ramathibodi Hospital, Mahidol University, Bangkok, Thailand. 


\section{Disclosure}

The authors report no conflicts of interest in this work.

\section{References}

1. Didona D, Maglie R, Eming R, Hertl M. Pemphigus: current and future therapeutic strategies. Front Immunol. 2019;10:1418.

2. Kasperkiewicz M, Ellebrecht CT, Takahashi H, et al. Pemphigus. Nat Rev Dis Primers. 2017;3:17026.

3. Kridin K, Sagi SZ, Bergman R. Mortality and cause of death in patients with pemphigus. Acta Derm Venereol. 2017;97(5):607-611. doi:10.2340/00015555-2611

4. Pollmann R, Schmidt T, Eming R, Hertl M. Pemphigus: a comprehensive review on pathogenesis, clinical presentation and novel therapeutic approaches. Clin Rev Allergy Immunol. 2018;54 (1): $1-25$.

5. Hammers CM, Stanley JR. Mechanisms of disease: pemphigus and bullous pemphigoid. Аnпи Rev Pathol. 2016;11(1):175-197. doi:10.1146/annurev-pathol-012615-044313

6. Bystryn JC. Adjuvant therapy of pemphigus. Arch Dermatol. 1984;120(7):941-951. doi:10.1001/archderm.1984.01650430127024

7. Bystryn JC, Steinman NM. The adjuvant therapy of pemphigus. An update. Arch Dermatol. 1996;132(2):203-212. doi:10.1001/ archderm.1996.03890260105016

8. Rice JB, White AG, Scarpati LM, Wan G, Nelson WW. Long-term systemic corticosteroid exposure: a systematic literature review. Clin Ther. 2017;39(11):2216-2229. doi:10.1016/j.clinthera.2017.09.011

9. Harman KE, Brown D, Exton LS, et al. British Association of Dermatologists' guidelines for the management of pemphigus vulgaris 2017. Br J Dermatol. 2017;177(5):1170-1201. doi:10.1111/ bjd. 15930

10. Tran KD, Wolverton JE, Soter NA. Methotrexate in the treatment of pemphigus vulgaris: experience in 23 patients. $\mathrm{Br} J$ Dermatol. 2013;169(4):916-921. doi:10.1111/bjd.12474

11. Sukanjanapong S, Thongtan D, Kanokrungsee S, Suchonwanit $P$, Chanprapaph K. A comparison of azathioprine and mycophenolate mofetil as adjuvant drugs in patients with pemphigus: a Retrospective Cohort Study. Dermatol Ther (Heidelb). 2020;10(1):179-189. doi:10.1007/s13555-019-00346-x

12. Sardana K, Agarwal P, Bansal S, Uppal B, Garg VK. A comparative effectiveness research of azathioprine and cyclophosphamide on the clinical and serological response in pemphigus vulgaris. Indian J Dermatol. 2016;61(4):418-426. doi:10.4103/0019-5154.185710

13. Thivolet J, Barthelemy H, Rigot-Muller G, Bendelac A. Effects of cyclosporin on bullous pemphigoid and pemphigus. Lancet. 1985;1 (8424):334-335. doi:10.1016/S0140-6736(85)91099-2

14. Segura S, Iranzo P, Martinez-de Pablo I, et al. High-dose intravenous immunoglobulins for the treatment of autoimmune mucocutaneous blistering diseases: evaluation of its use in 19 cases. $\mathrm{J} \mathrm{Am} \mathrm{Acad}$ Dermatol. 2007;56(6):960-967. doi:10.1016/j.jaad.2006.06.029

15. Ruocco E, Wolf R, Ruocco V, Brunetti G, Romano F, Lo Schiavo A. Pemphigus: associations and management guidelines: facts and controversies. Clin Dermatol. 2013;31(4):382-390. doi:10.1016/j. clindermatol.2013.01.005

16. Svecova D. IVIG therapy in pemphigus vulgaris has corticosteroid-sparing and immunomodulatory effects. Australas J Dermatol. 2016;57(2):141-144. doi:10.1111/ajd.12422

17. Roujeau JC, Andre C, Joneau Fabre M, et al. Plasma exchange in pemphigus. Uncontrolled study of ten patients. Arch Dermatol. 1983;119(3):215-221. doi:10.1001/archderm.1983.01650270033013

18. Feldman RJ, Ahmed AR. Relevance of rituximab therapy in pemphigus vulgaris: analysis of current data and the immunologic basis for its observed responses. Expert Rev Clin Immunol. 2011;7 (4):529-541. doi:10.1586/eci.11.22
19. Colliou N, Picard D, Caillot F, et al. Long-term remissions of severe pemphigus after rituximab therapy are associated with prolonged failure of desmoglein B cell response. Sci Transl Med. 2013;5 (175):175ra130. doi:10.1126/scitranslmed.3005166

20. Biogen Inc., Inc. G. Rituxan ${ }^{\circledR}$ (Rituximab) US prescribing information [updated 01/2019]; 1997.

21. Cianchini G, Lupi F, Masini C, Corona R, Puddu P, De Pita O. Therapy with rituximab for autoimmune pemphigus: results from a single-center observational study on 42 cases with long-term follow-up. J Am Acad Dermatol. 2012;67(4):617-622. doi:10.1016/ j.jaad.2011.11.007

22. Leshem YA, Hodak E, David M, Anhalt GJ, Mimouni D. Successful treatment of pemphigus with biweekly 1-g infusions of rituximab: a retrospective study of 47 patients. $J$ Am Acad Dermatol. 2013;68 (3):404-411. doi:10.1016/j.jaad.2012.08.010

23. Ahmed AR, Spigelman Z, Cavacini LA, Posner MR. Treatment of pemphigus vulgaris with rituximab and intravenous immune globulin. N Engl J Med. 2006;355(17):1772-1779. doi:10.1056/NEJMoa062930

24. Joly P, Maho-Vaillant M, Prost-Squarcioni C, et al. First-line rituximab combined with short-term prednisone versus prednisone alone for the treatment of pemphigus (Ritux 3): a prospective, multicentre, parallel-group, open-label randomised trial. Lancet. 2017;389 (10083):2031-2040. doi:10.1016/S0140-6736(17)30070-3

25. Joly P, Horwath B, Patsatsi A, et al. Updated S2K guidelines on the management of pemphigus vulgaris and foliaceus initiated by the european academy of dermatology and venereology (EADV). $J$ Eur Acad Dermatol Venereol. 2020;34(9):1900-1913. doi:10.1111/ jdv. 16752

26. Murrell DF, Pena S, Joly P, et al. Diagnosis and management of pemphigus: recommendations of an international panel of experts. J Am Acad Dermatol. 2020;82(3):575-585 e571. doi:10.1016/j. jaad.2018.02.021

27. Shimizu T, Takebayashi T, Sato Y, et al. Grading criteria for disease severity by pemphigus disease area index. J Dermatol. 2014;41 (11):969-973. doi:10.1111/1346-8138.12649

28. Rosenbach M, Murrell DF, Bystryn JC, et al. Reliability and convergent validity of two outcome instruments for pemphigus. $J$ Invest Dermatol. 2009;129(10):2404-2410. doi:10.1038/jid.2009.72

29. Kim TH, Choi Y, Lee SE, Lim JM, Kim SC. Adjuvant rituximab treatment for pemphigus: a retrospective study of 45 patients at a single center with long-term follow up. J Dermatol. 2017;44 (6):615-620. doi:10.1111/1346-8138.13757

30. Murrell DF, Dick S, Ahmed AR, et al. Consensus statement on definitions of disease, end points, and therapeutic response for pemphigus. $\quad J$ Am Acad Dermatol. 2008;58(6):1043-1046. doi:10.1016/j.jaad.2008.01.012

31. Vinay K, Cazzaniga S, Amber KT, Feldmeyer L, Naldi L, Borradori L. Rituximab as first-line adjuvant therapy for pemphigus: retrospective analysis of long-term outcomes at a single center. $J \mathrm{Am}$ Acad Dermatol. 2018;78(4):806-808. doi:10.1016/j.jaad.2017.11.024

32. Hebert V, Vermeulin T, Tanguy L, et al. Comparison of real costs in the French healthcare system in newly diagnosed patients with pemphigus for first-line treatment with rituximab vs. standard corticosteroid regimen: data from a national multicentre trial. $\mathrm{Br} J$ Dermatol. 2020;183(1):121-127. doi:10.1111/bjd.18563

33. Sharma VK, Gupta V, Bhari N, Singh V. Rituximab as an adjuvant therapy for pemphigus: experience in 61 patients from a single center with long-term follow-up. Int J Dermatol. 2020;59(1):76-81.

34. Kurihara Y, Yamagami J, Funakoshi T, et al. Rituximab therapy for refractory autoimmune bullous diseases: a multicenter, open-label, single-arm, Phase $1 / 2$ study on 10 Japanese patients. J Dermatol. 2019;46(2):124-130. doi:10.1111/1346-8138.14732

35. Kushner CJ, Wang S, Tovanabutra N, Tsai DE, Werth VP, Payne AS. Factors associated with complete remission after rituximab therapy for pemphigus. JAMA Dermatol. 2019;155(12):1404-1409. doi:10.1001/jamadermatol.2019.3236 
36. Chen DM, Odueyungbo A, Csinady E, et al. Rituximab is an effective treatment in patients with pemphigus vulgaris and demonstrates a steroid-sparing effect. $\mathrm{Br} \quad J$ Dermatol. 2020;182(5):1111-1119. doi:10.1111/bjd.18482

37. De D, Bishnoi A, Handa S, Mahapatra T, Mahajan R. Effectiveness and safety analysis of rituximab in 146 Indian pemphigus patients: a retrospective single-center review of up to 68 months follow-up. Indian J Dermatol Venereol Leprol. 2020;86(1):39-44. doi:10.4103/ ijdvl.IJDVL $848 \quad 17$

38. Balighi K, Daneshpazhooh M, Akbari Z, Tavakolpour S, Azimi P, Azizpour A. Comparing the short-term therapeutic effects and safety profiles of rituximab therapy in pemphigus vulgaris patients either early treated or later than six months. J Dermatolog Treat. 2019;30 (4):346-349. doi:10.1080/09546634.2018.1509049

39. Balighi K, Daneshpazhooh M, Mahmoudi H, et al. Comparing early and late treatments with rituximab in pemphigus vulgaris: which one is better? Arch Dermatol Res. 2019;311(1):63-69. doi:10.1007/ s00403-018-1881-1

40. Lunardon L, Tsai KJ, Propert KJ, et al. Adjuvant rituximab therapy of pemphigus: a single-center experience with 31 patients. Arch Dermatol. 2012;148(9):1031-1036. doi:10.1001/archdermatol. 2012.1522

41. Hammers CM, Chen J, Lin C, et al. Persistence of anti-desmoglein 3 $\mathrm{IgG}(+)$ B-cell clones in pemphigus patients over years. $J$ Invest Dermatol. 2015;135(3):742-749. doi:10.1038/jid.2014.291

42. Schmidt E. Rituximab as first-line treatment of pemphigus. Lancet. 2017;389(10083):1956-1958. doi:10.1016/S0140-6736(17)30787-0

43. Suchonwanit P, Hector CE, Bin Saif GA, McMichael AJ. Factors affecting the severity of central centrifugal cicatricial alopecia. Int J Dermatol. 2016;55(6):e338-343. doi:10.1111/ijd.13061

44. Cho YT, Lee FY, Chu CY, Wang LF. First-line combination therapy with rituximab and corticosteroids is effective and safe for pemphigus. Acta Derm Venereol. 2014;94(4):472-473. doi:10.2340/ 00015555-1746

45. Cho YT, Huang YM, Wang LF, Chu CY. Maintenance therapy with azathioprine prolonged duration of remission for pemphigus patients who received rituximab as first-line or add-on therapy. J Formos Med Assoc. 2020;119(1 Pt 2):230-237. doi:10.1016/j.jfma.2019.04.020

46. Hertl M, Jedlickova H, Karpati S, et al. Pemphigus. S2 Guideline for diagnosis and treatment-guided by the European Dermatology Forum (EDF) in cooperation with the European Academy of Dermatology and Venereology (EADV). J Eur Acad Dermatol Venereol. 2015;29 (30):405-414. doi:10.1111/jdv.12772

47. Amber KT, Hertl M. An assessment of treatment history and its association with clinical outcomes and relapse in 155 pemphigus patients with response to a single cycle of rituximab. J Eur Acad Dermatol Venereol. 2015;29(4):777-782. doi:10.1111/jdv.12678
48. Wang HH, Liu CW, Li YC, Huang YC. Efficacy of rituximab for pemphigus: a systematic review and meta-analysis of different regimens. Acta Derm Venereol. 2015;95(8):928-932. doi:10.2340/ 00015555-2116

49. Tavakolpour S, Mahmoudi H, Balighi K, Abedini R, Daneshpazhooh M. Sixteen-year history of rituximab therapy for 1085 pemphigus vulgaris patients: a systematic review. Int Immunopharmacol. 2018;54:131-138. doi:10.1016/j.intimp.2017. 11.005

50. Keren Z, Naor S, Nussbaum S, et al. B-cell depletion reactivates $\mathrm{B}$ lymphopoiesis in the BM and rejuvenates the B lineage in aging. Blood. 2011;117(11):3104-3112. doi:10.1182/blood-2010-09-307983

51. Harman KE, Seed PT, Gratian MJ, Bhogal BS, Challacombe SJ, Black MM. The severity of cutaneous and oral pemphigus is related to desmoglein 1 and 3 antibody levels. Br J Dermatol. 2001;144 (4):775-780. doi:10.1046/j.1365-2133.2001.04132.x

52. Eming R, Hertl M. Immunoadsorption in pemphigus. Autoimmunity. 2006;39(7):609-616. doi:10.1080/08916930600972040

53. Abasq C, Mouquet H, Gilbert D, et al. ELISA testing of anti-desmoglein 1 and 3 antibodies in the management of pemphigus. Arch Dermatol. 2009;145(5):529-535. doi:10.1001/ archdermatol.2009.9

54. Albers LN, Liu Y, Bo N, Swerlick RA, Feldman RJ. Developing biomarkers for predicting clinical relapse in pemphigus patients treated with rituximab. J Am Acad Dermatol. 2017;77(6):1074-1082. doi:10.1016/j.jaad.2017.07.012

55. Saleh MA. A prospective study comparing patients with early and late relapsing pemphigus treated with rituximab. $J \mathrm{Am}$ Acad Dermatol. 2018;79(1):97-103. doi:10.1016/j.jaad.2018.01.029

56. Mignard C, Maho-Vaillant M, Golinski ML, et al. Factors associated with short-term relapse in patients with pemphigus who receive rituximab as first-line therapy: a post hoc analysis of a Randomized Clinical Trial. JAMA Dermatol. 2020;156(5):545-552. doi:10.1001/ jamadermatol.2020.0290

57. Ahmed AR, Shetty S. A comprehensive analysis of treatment outcomes in patients with pemphigus vulgaris treated with rituximab. Autoimmun Rev. 2015;14(4):323-331. doi:10.1016/j.autrev.2014. 12.002

58. Heelan K, Al-Mohammedi F, Smith MJ, et al. Durable remission of pemphigus with a fixed-dose rituximab protocol. JAMA Dermatol. 2014;150(7):703-708. doi:10.1001/jamadermatol.2013.6739

59. Anandan V, Jameela WA, Sowmiya R, Kumar MMS, Lavanya P. Rituximab: a magic bullet for pemphigus. J Clin Diagn Res. 2017;11 (4):WC01-WC06. doi:10.7860/JCDR/2017/21868.9717

\section{Publish your work in this journal}

Drug Design, Development and Therapy is an international, peerreviewed open-access journal that spans the spectrum of drug design and development through to clinical applications. Clinical outcomes, patient safety, and programs for the development and effective, safe, and sustained use of medicines are a feature of the journal, which has also been accepted for indexing on PubMed Central. The manuscript management system is completely online and includes a very quick and fair peer-review system, which is all easy to use. Visit http://www. dovepress.com/testimonials.php to read real quotes from published authors. 